\title{
Exceptionally Long-Lived Photodriven Multi-Electron Storage without Sacrificial Reagents
}

\author{
Martin Kuss-Petermann, ${ }^{[a]}$ and Oliver S. Wenger ${ }^{*[a]}$
}

\begin{abstract}
Photo-excitation of a molecular pentad in presence of $\mathrm{Sc}^{3+}$ in de-aerated $\mathrm{CH}_{3} \mathrm{CN}$ leads to a quinone dianion that is stable on the millisecond timescale. Light-driven electron accumulation on the quinone unit is sensitized by two $\mathrm{Ru}(\mathrm{bpy})_{3}{ }^{2+}$ complexes in an intramolecular process, which relies on covalently attached triarylamine donors rather than on sacrificial reagents. Lewis acid Lewis base interactions between $\mathrm{Sc}^{3+}$ and quinone dianion are responsible for the exceptionally long lifetime of this photoproduct. Our study of photo-induced multi-electron transfer is relevant in the greater context of solar energy conversion.
\end{abstract}

\section{Introduction}

Because of the light-driven accumulation of redox equivalents, the $\mathrm{Mn}_{4} \mathrm{Ca}$ cluster of photosystem II is able to oxidize water. ${ }^{[1]}$ Similarly, the accumulation of reductive equivalents is important for the activation of small inert molecules such as $\mathrm{CO}_{2}$ or $\mathrm{N}_{2}$. Much effort has gone into the development of artificial water oxidation and $\mathrm{CO}_{2}$ reduction catalysts in recent years, and impressive progress has been made. ${ }^{[2]}$ However, in the vast majority of cases, sacrificial redox reagents were employed, and this is partly owed to the fact that the elementary principles of photoinduced multi-electron transfer are not nearly as well understood as those of single electron transfer. Sustainable solar energy conversion will not be possible with sacrificial reagents, ${ }^{[3]}$ and therefore it is desirable to explore the basics of photoinduced charge accumulation.

The investigation of covalently linked donor-bridge-acceptor molecules has been very useful for understanding the basic principles of single electron transfer ${ }^{[4]}$ for example through driving-force variations, ${ }^{[5]}$ distance dependence studies, ${ }^{[6]}$ or bridge alterations. ${ }^{[7]}$ Later, combined electron and proton transfer reactions were explored using the same donor-bridgeacceptor approach, ${ }^{[8]}$ giving insight into proton-coupled electron transfer (PCET). For exploring photoinduced charge accumulation, donor-bridge-acceptor systems are promising as well, but at present, only a handful of suitable compounds is known. ${ }^{[9]}$ Two early studies reported on intramolecular electron accumulation in molecular triads, but the lifetimes of the photoproducts were restricted to $5 \mathrm{~ns} .{ }^{[10]}$ In a related early study,

[a] Dr. M. Kuss-Petermann, Prof. Dr. O. S. Wenger Department of Chemistry

University of Basel

St. Johanns-Ring 19, 4056 Basel (Switzerland)

E-mail: oliver.wenger@unibas.ch

Supporting information for this article is given via a link at the end of the document. a very short excitation wavelength $(266 \mathrm{~nm})$ had to be used to generate a short-lived accumulated electron pair. ${ }^{[11]}$ Later, a whole series of compounds in which redox equivalents can be accumulated with visible light was reported, but these systems all relied on sacrificial reagents. ${ }^{[12]}$ Only relatively recently, accumulation of oxidative equivalents without the use of sacrificial reagents has been achieved, albeit in a system with $\mathrm{TiO}_{2}$ nanoparticles. ${ }^{[13]}$ We recently communicated molecular pentad I (Scheme 1), ${ }^{[14]}$ which is the first purely molecular system in which long-lived (> $5 \mathrm{~ns}$ ) electron accumulation is observed without sacrificial reagents. In pentad I, electron accumulation is an entirely intramolecular process in which the two covalently attached triarylamine (TAA) units act as (reversible) one-electron donors, whereas the central anthraquinone (AQ) acceptor stores two electrons for $870 \mathrm{~ns}$ in de-aerated $\mathrm{CH}_{3} \mathrm{CN}$ at $20^{\circ} \mathrm{C}$.

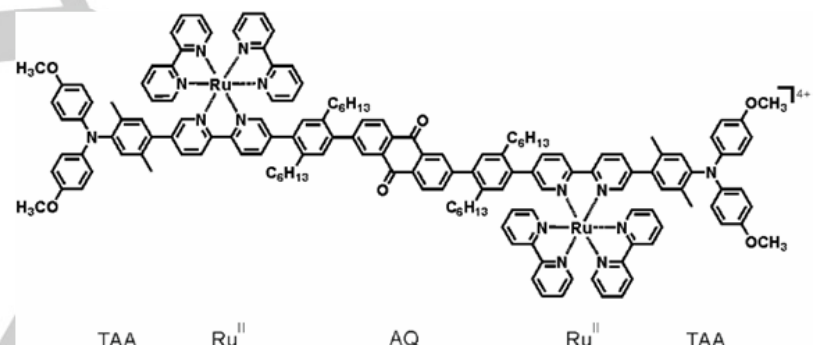

Scheme 1. Molecular structure of pentad I and labeling of its individual components.

It seems worthwhile to stress how important the conceptual difference between the covalently attached triarylamine donors in pentad $\mathbf{I}$ and the use of sacrificial, non-covalently attached electron donors in prior studies is. Sacrificial donors decompose after electron donation, making undesired re-oxidation of the substrate an essentially irreversible process. By contrast, as noted above, triarylamines are reversible donors that do not decompose, and hence undesired reverse electron transfers can readily occur after initial charge-separation. In fact the oxidized triarylamine is a strong electron acceptor that can easily impede electron accumulation at the central acceptor site due to large driving-force and due to covalent attachment. ${ }^{[9 a}$, 15] With sacrificial quenchers this is far less problematic because they decompose to poor electron acceptors and moreover they diffuse away after initial electron transfer, leading to spatial separation between electrons and holes. Thus it is clear that obtaining electron accumulation in intramolecular fashion by using reversible electron donors is far more challenging than using non-covalently attached sacrificial donors. 
While our initial studies were conducted in neat $\mathrm{CH}_{3} \mathrm{CN}$ leading to electron accumulation at anthraquinone, subsequent further investigations provided unprecedented mechanistic insight into multi-electron, multi-proton chemistry by studying the photophysics and the photochemistry of pentad I in presence of p-toluenesulfonic acid. ${ }^{[16]}$ However, the lifetime of the chargeaccumulated state was only extended from $870 \mathrm{~ns}$ to $4.7 \mu \mathrm{s}$ in presence of Bronsted acid. [16]

In this work, we sought to stabilize the two electrons on $A Q$ even further by exploiting metal ion-coupled electron transfer (MCET) ${ }^{[17]}$ Prior studies have shown that Lewis acids such as $\mathrm{Sc}^{3+}$ or $\mathrm{Y}^{3+}$ can stabilize quinone radical anions, ${ }^{[18]}$ and we hypothesized that Lewis acid - Lewis base interactions could also be useful for extending the lifetime of our photogenerated anthraquinone dianion species. This is indeed the case, and herein we demonstrate that MCET gives access to a chargeaccumulated state with a lifetime in the millisecond regime, more than 3 orders of magnitude longer than in neat $\mathrm{CH}_{3} \mathrm{CN}$. Thus, our system is able to store redox equivalents on the same timescale as the oxygen-evolving complex of photosystem II. ${ }^{[19]}$

\section{Results and Discussion}

In order to detect photoinduced charge accumulation unambiguously, the various redox states of a system must be spectroscopically clearly distinguishable from one another. In prior work we demonstrated that this is the case for anthraquinone $(\mathrm{AQ})$ in neat $\mathrm{CH}_{3} \mathrm{CN}$ in the infrared spectral region, and we used transient IR studies to distinguish $A Q^{2-}$ from $A Q^{-}$in pentad $I^{[14]}$ In the present study, we explored charge accumulation in presence of the strong Lewis acid $\mathrm{Sc}^{3+}$ with UVVis transient absorption spectroscopy. As demonstrated in the following, the spectroscopic signatures of the various redox states of $A Q$ in presence of $S^{3+}$ resemble those of $A Q$ in presence of Bronsted acids, and this forms the basis for unambiguous detection of doubly reduced $\mathrm{AQ}$ with $\mathrm{Sc}^{3+}$ bound to it.

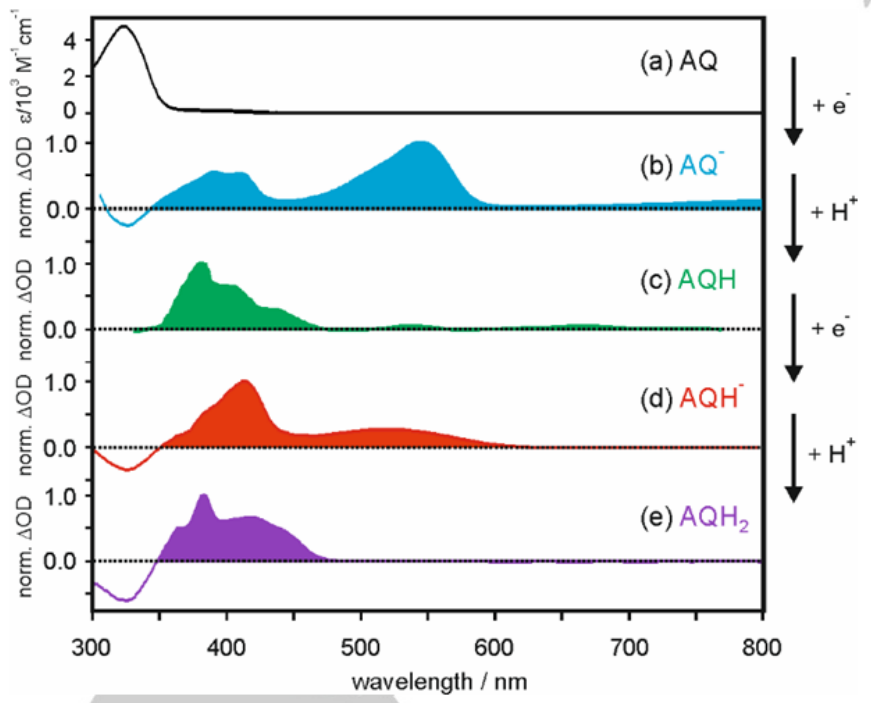

Figure 1. (a) UV-Vis absorption spectrum of 9,10-anthraquinone (AQ) in $\mathrm{CH}_{3} \mathrm{CN}$. The spectra in (b) - (e) are differential absorption spectra, i. e., they show the spectral changes associated with the conversion of a charge-neutral anthraquinone species into various reduced and protonated forms thereof. (b)
Difference spectrum of 9,10-anthraquinone radical anion (AQ-) in DMF. ${ }^{[20]}$ (c) Difference spectrum of the semiquinone form $(A Q H)$ of 2-hydroxy-9,10anthraquinone in water / isopropanol / acetone (30:5:1, v:v:v) mixture. ${ }^{[21]}(\mathrm{d})$ Difference spectrum of the hydroquinone anion form $\left(\mathrm{AQH}^{-}\right)$of $9,10-$ anthraquinone in DMF. ${ }^{[20]}$ (e) Difference spectrum of the hydroquinone form $\left(\mathrm{AQH}_{2}\right)$ of 9,10 -anthraquinone in DMF. ${ }^{[20]}$

$A Q$ does not absorb significantly at wavelengths longer than 370 $\mathrm{nm}$ (Figure 1a), but upon reduction to $\mathrm{AQ}^{-}$bands at 390,410 , and $545 \mathrm{~nm}$ emerge (Figure $1 \mathrm{~b}$ ). ${ }^{[20]}$ Protonation of $A Q^{-}$to afford the semiquinone $A Q H$ leads to disappearance of the $545 \mathrm{~nm}$ band while absorption in the range from 370 to $420 \mathrm{~nm}$ persists (Figure 1c). ${ }^{[21-22]}$ Further reduction to the hydroquinone anion $\mathrm{AQH}^{-}$(doubly reduced and singly protonated $\mathrm{AQ}$ ) causes absorptions centered at 412 and $520 \mathrm{~nm}$ (Figure 1d). ${ }^{[20]}$ Finally, protonation of $\mathrm{AQH}^{-}$to yield the neutral hydroquinone form $\mathrm{AQH}_{2}$ leads to absorptions exclusively below $480 \mathrm{~nm}$ with a distinct narrow feature at $\sim 380 \mathrm{~nm}$ (Figure 1e). ${ }^{[20]}$

For UV-Vis transient absorption spectroscopy, $20 \mu \mathrm{M}$ solutions of pentad $\mathrm{I}$ in de-aerated $\mathrm{CH}_{3} \mathrm{CN}$ in presence of $50 \mathrm{mM} \mathrm{Sc}(\mathrm{OTf})_{3}$ were excited at $532 \mathrm{~nm}$ with pulses of $\sim 10 \mathrm{~ns}$ duration. Since the focus of this study was on comparatively slow processes, experiments with shorter excitation pulses were not undertaken. Kinetic decays were recorded in $5 \mathrm{~nm}$ intervals between 320 and $800 \mathrm{~nm}$, and subsequently the data were fitted globally to result in species-associated difference spectra (SADS). ${ }^{[23]}$ The initial spectrum at $\mathrm{t}=0$ exhibits bands with maxima at 375,585 , and $770 \mathrm{~nm}$ (black trace in Figure 2a). The best global fits to this data set consisting of 97 decays in $20 \mu$ s time windows were obtained using a bi-exponential function with a residual, which reflects signals that persist for over $20 \mu$ s. The first SADS (blue trace in Figure 2a) has an associated lifetime of $170 \mathrm{~ns}$ and exhibits absorptions at 365 and $585 \mathrm{~nm}$, as well as a negative signal at $410 \mathrm{~nm}$. The bands at 365 and $585 \mathrm{~nm}$ are due to $A Q$ (Figure 1b); in fact we and others have seen these two specific absorptions in many donor-bridge-acceptor compounds with $A Q$ before. ${ }^{[6 f, 24]}$ Thus, the positive signals at 365 and $585 \mathrm{~nm}$ indicate that $A Q^{-}$disappears, whereas the negative signal at 410 $\mathrm{nm}$ indicates the formation of a new species with stronger extinction at this wavelength. It is plausible that this new species is the $\mathrm{AQ}^{-} / \mathrm{Sc}^{3+}$ adduct, which should spectroscopically resemble $A Q H$ (Figure 1c). 


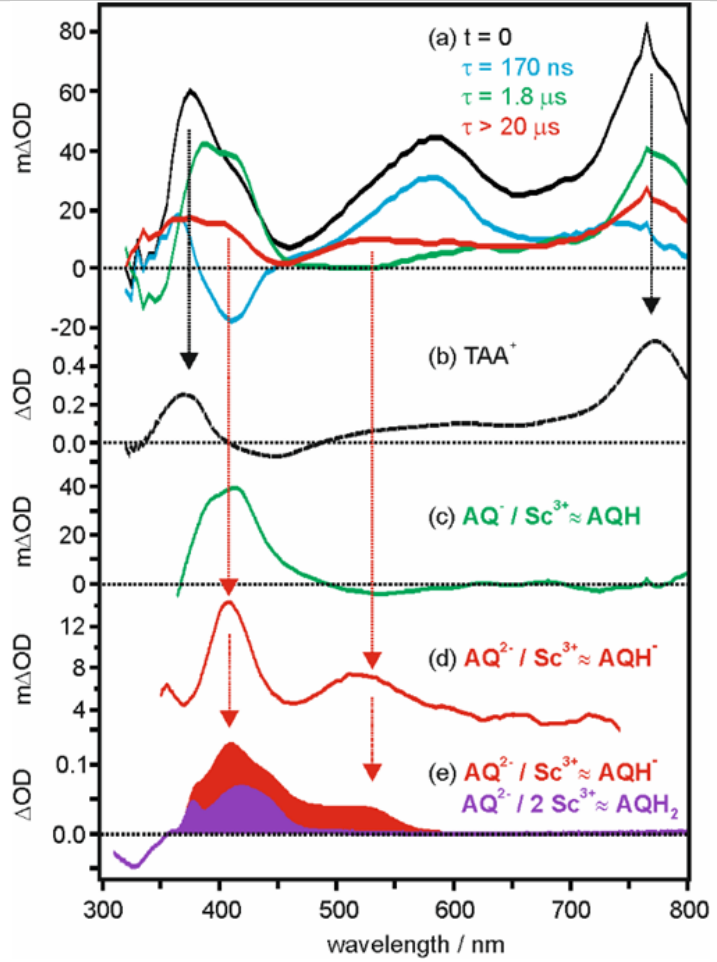

Figure 2. (a) Transient absorption spectra obtained from a de-aerated $\mathrm{CH}_{3} \mathrm{CN}$ solution of $20 \mu \mathrm{M}$ pentad I in presence of $50 \mathrm{mM} \mathrm{Sc}(\mathrm{OTf})_{3}$ at $20{ }^{\circ} \mathrm{C}$. Excitation was at $532 \mathrm{~nm}$ with laser pulses of $\sim 10 \mathrm{~ns}$ duration. Black trace: Initial spectrum at $\mathrm{t}=0$. Blue, green, red traces: SADS with decay times as indicated in the inset. (b) Differential absorption spectrum showing the spectral changes associated with oxidation of TAA in pentad $\mathbf{I}$, obtained by spectroelectrochemistry in $\mathrm{CH}_{3} \mathrm{CN}$ (applied potential: $0.8 \mathrm{~V}$ vs. SCE). (c) Contribution of reduced $A Q$ species to the overall $1.8 \mu \mathrm{s}-\mathrm{SADS}$, obtained by subtracting the appropriately scaled spectrum from (b) from the green trace in (a); the resulting spectrum is similar to that of $A Q H$ in Figure 1c. (d) Contribution of reduced $A Q$ species to the overall $>20 \mu \mathrm{s}-\mathrm{SADS}$, obtained by subtracting the appropriately scaled spectrum from (b) from the red trace in (a); the resulting spectrum is similar to that of $\mathrm{AQH}^{-}$in Figure 1d. (e) Differential absorption spectra obtained in the course of applying a potential of $-1.6 \mathrm{~V}$ vs. SCE to a solution containing $0.5 \mathrm{mM} \mathrm{AQ}, 0.5 \mathrm{mM} \mathrm{Sc}(\mathrm{OTf})_{3}$ and $0.1 \mathrm{M} \mathrm{TBAPF}_{6}$ in $\mathrm{CH}_{3} \mathrm{CN}$; the spectrum obtained prior to applying the potential served as a baseline. The initially recorded purple signature resembles the spectrum of $\mathrm{AQH}_{2}$ in Figure 1e, while the red signature measured later resembles the spectrum of $\mathrm{AQH}^{-}$in Figure 1d.

The second SADS (green trace in Figure 2a) has an associated lifetime of $1.8 \mu \mathrm{s}$, and it exhibits absorption maxima at 390, 410, and $770 \mathrm{~nm}$. The band at $770 \mathrm{~nm}$ is caused by the triarylamine radical cation $\left(\mathrm{TAA}^{+}\right),{ }^{[6 f, 24 \mathrm{e}, 25]}$ and the difference spectrum of $\mathrm{TAA}^{+}$in pentad $\mathbf{I}$ is shown in Figure $2 \mathrm{~b}$ (dashed black trace). When scaling the $\mathrm{TAA}^{+}$difference spectrum such that it matches the $\triangle \mathrm{OD}$ in the $1.8 \mu \mathrm{s}$-SADS at $770 \mathrm{~nm}$ (green trace in Figure $2 \mathrm{a}$ ) and then subtracting it, the green trace in Figure $2 \mathrm{c}$ is obtained. This green spectrum represents the contribution of reduced anthraquinone species to the overall $1.8 \mu \mathrm{s}-\mathrm{SADS}$. The respective spectrum resembles that of the semiquinone $A Q H$ (Figure 1c), suggesting that the observed photoproduct is indeed the $\mathrm{AQ}^{-} / \mathrm{Sc}^{3+}$ adduct anticipated above. The negative signal at $410 \mathrm{~nm}$ in the $170 \mathrm{~ns}-\mathrm{SADS}$ (blue trace in Figure 2a) is compatible with the formation of this adduct.

The global fit to the transient absorption data yields a residual signal with a lifetime longer than $20 \mu \mathrm{s}$, exhibiting the spectral signature represented by the red trace in Figure 2a. When subtracting the contribution of $\mathrm{TAA}^{+}$from this spectrum, the red trace in Figure $2 \mathrm{~d}$ is obtained. This spectrum exhibits absorption maxima at 410 and $515 \mathrm{~nm}$, and its overall appearance is very similar to the spectrum of $\mathrm{AQH}^{-}$(Figure 1d). Spectroelectrochemical studies performed with $A Q$ in presence of $\mathrm{Sc}(\mathrm{OTf})_{3}$ result in the spectra shown in Figure 2e. The red signature strongly resembles the spectrum in Figure $2 d$ and the signature of $\mathrm{AQH}^{-}$(Figure 1d), and consequently is attributed to the $\mathrm{AQ}^{2-} / \mathrm{Sc}^{3+}$ adduct. For these spectro-electrochemical experiments, a potential of $-1.6 \mathrm{~V}$ vs. SCE was applied to a solution containing $5 \mathrm{mM} \mathrm{9,10-anthraquinone,} 5 \mathrm{mM} \mathrm{Sc}(\mathrm{OTf})_{3}$, and $0.1 \mathrm{M} \mathrm{TBAPF}_{6}$ in $\mathrm{CH}_{3} \mathrm{CN}$. At that potential, $\mathrm{AQ}$ is reduced to $A Q^{2-}{ }^{[14]}$ and initially one obtains the purple signature in Figure 2e. With its absorption band around $420 \mathrm{~nm}$ and a distinct narrow feature at $\sim 380 \mathrm{~nm}$ this purple signature is very similar to the spectrum of $\mathrm{AQH}_{2}$ (purple trace in Figure 1e), and consequently is attributed to the $\mathrm{AQ}^{2-} / 2 \mathrm{Sc}^{3+}$ adduct, i. e., doubly reduced anthraquinone with two $\mathrm{Sc}^{3+}$ cations bound to it. In the course of reducing increasing amounts of $A Q$ to $A Q^{2-}$, the purple spectrum converts to the red spectrum in Figure 2e, which we attributed above to the $\mathrm{AQ}^{2-} / \mathrm{Sc}^{3+}$ (1:1) adduct. This shift in equilibrium from $1: 2$ to $1: 1$ adducts evidently occurs because at some point there is not enough Lewis acid present in solution to allow binding of $2 \mathrm{Sc}^{3+}$ cations per electrochemically generated $A^{2-}$ species. When using higher concentrations of $\mathrm{Sc}^{3+}$ (20 equivalents), only the 1:2 adduct is observed.

The $\mathrm{AQ}^{2-} / \mathrm{Sc}^{3+}(1: 1)$ adduct exhibits a diagnostic absorption at $515 \mathrm{~nm}$ (red traces in Figure 2a/d) that is absent in any of the other SADS (blue and green traces in Figure 2a), indicating that this signal is present from the very beginning. In other words, the $\mathrm{AQ}^{2-} / \mathrm{Sc}^{3+}$ species is formed within the $\sim 10 \mathrm{~ns}$ duration of the laser excitation pulses, and then exhibits a very slow (>20 $\mu \mathrm{s})$ decay. At a pentad concentration of $20 \mu \mathrm{M}$, such rapid charge accumulation is only viable in intramolecular fashion.

The extinction coefficient at $515 \mathrm{~nm}$ could not be determined reliably, but the relative amount of $\mathrm{AQ}^{2-} / \mathrm{Sc}^{3+}$ adduct formed can be estimated on the basis of the $\triangle O D$ values at $770 \mathrm{~nm}$. At that wavelength, $\mathrm{TAA}^{+}$is the only absorbing species (see above). When taking into account that the formation of $\mathrm{AQ}^{2-} / \mathrm{Sc}^{3+}$ implies the presence of two $\mathrm{TAA}^{+}$units per pentad molecule, comparison of the optical densities at $770 \mathrm{~nm}$ in the red and the black traces in Figure 2 a leads to the conclusion that $19 \%$ of the observable photoproducts are charge accumulated species, whereas the remaining $\sim 81 \%$ are due to the $\mathrm{AQ}^{-}$and $\mathrm{AQ}^{-} / \mathrm{Sc}^{3+}$ species discussed above. We note that this product ratio is expected to be excitation power-dependent, ${ }^{[14]}$ and the values reported here were obtained using pulse energies of $18 \mathrm{~mJ}$. In our prior communication, ${ }^{[14]}$ we demonstrated that the concentration of charge accumulated species formed depends in quadratic fashion on the excitation power, at least at very low excitation powers. Such behavior is expected for a two-photon process. 


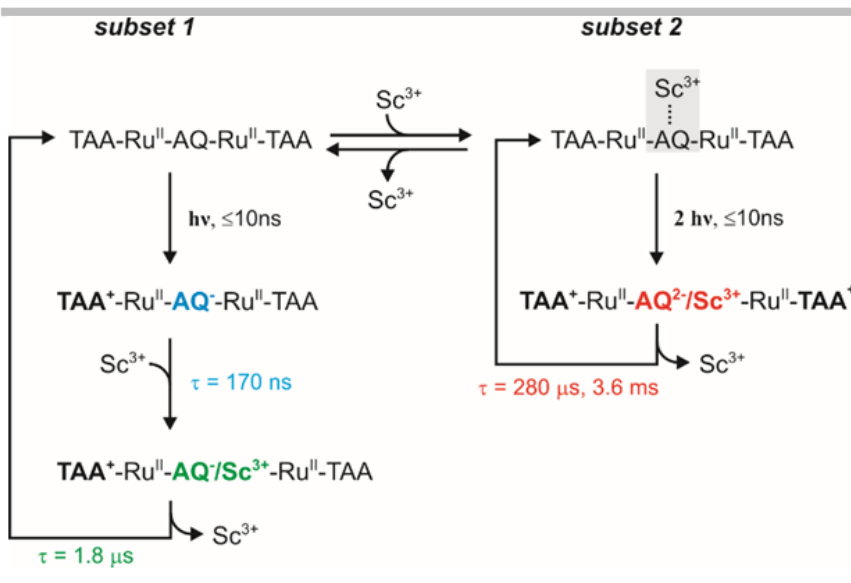

Scheme 2. Reaction sequences leading to the observable $A Q^{-}, A Q^{-} / \mathrm{Sc}^{3+}$, and $\mathrm{AQ}^{2-} / \mathrm{Sc}^{3+}$ photoproducts after excitation of pentad $\mathrm{I}$ in presence of $\mathrm{Sc}^{3+}$.

It seems plausible that the different photoproducts are formed in the fashion illustrated in Scheme 2. A first subset of pentad molecules has no associated $\mathrm{Sc}^{3+}$ cations when it is photoexcited and undergoes rapid ( $<50 \mathrm{ps)}$ charge separation to produce a $\mathrm{TAA}^{+} / \mathrm{AQ}^{-}$radical ion pair by pathways similar to those explored previously in simpler compounds. ${ }^{[24,}$ 26] $\mathrm{Sc}^{3+}$ cations then bind to this photoproduct with a time constant of $170 \mathrm{~ns}$ (blue trace in Figure 2a), and the resulting $\mathrm{AQ}^{-} / \mathrm{Sc}^{3+}$ adduct subsequently decays with a time constant of $1.8 \mu \mathrm{s}$ (green trace in Figure 2a). A second subset of pentad molecules (Scheme 2, right) has weakly associated $\mathrm{Sc}^{3+}$ cations when it is excited, and then undergoes rapid ( $<10 \mathrm{~ns}$ ) intramolecular charge accumulation to form $\mathrm{AQ}^{2-} / \mathrm{Sc}^{3+}$ and two $\mathrm{TAA}^{+}$units (red trace in Figure 2a). This photoproduct exhibits a lifetime $>20 \mu \mathrm{s}$, due to stabilization of quinone dianion by the strong Lewis acid $\mathrm{Sc}^{3+}$ (see below). Absorption of two photons by pentad molecules without associated $\mathrm{Sc}^{3+}$ cations does not lead to an observable long-lived charge accumulated state.

Two reference experiments were performed to corroborate this interpretation. First, we replaced $\mathrm{Sc}^{3+}$ by an equal concentration of the much weaker Lewis acid $\mathrm{Ca}^{2+}$. No evidence for an $\mathrm{AQ}^{2-}$, $\mathrm{Ca}^{2+}$ adduct is found (Supporting Information, Figure S1), indicating that the charge-accumulated species either does not form at all, or does not live long enough to be detectable on top of the more intense signal from the $A Q^{-\cdots} \mathrm{Ca}^{2+}$ product. The transient absorption spectrum of the observed reduced species closely resembles the signature of hydrogen-bonded $\mathrm{AQ}^{-}$(Figure S2). Very likely, a long-lived charge accumulated state is inaccessible due to lack of significant pre-association between $\mathrm{Ca}^{2+}$ and $\mathrm{AQ}$ prior to photo-excitation. In the second reference experiment, the $\mathrm{Sc}^{3+}$ concentration was lowered from 50 to 5 $\mathrm{mM}$ (Figure S3). All photoproducts identified above remained detectable, but the decay of $A Q^{-}$at the expense of the formation of $\mathrm{AQ}^{-} / \mathrm{Sc}^{3+}$ was now slower (470 instead of $170 \mathrm{~ns}$ ), and the relative amount of $\mathrm{AQ}^{2-} / \mathrm{Sc}^{3+}$ was now only $4 \%$ instead of $19 \%$. These observations are all compatible with the view outlined in Scheme 2.

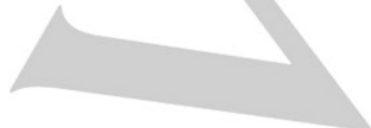

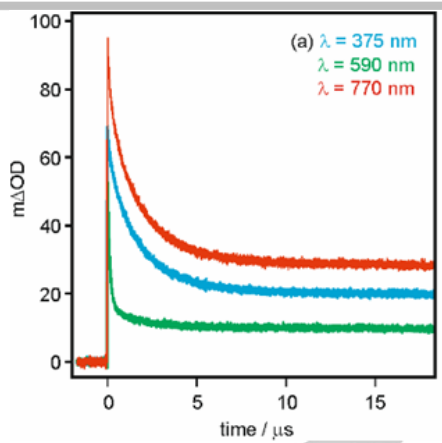

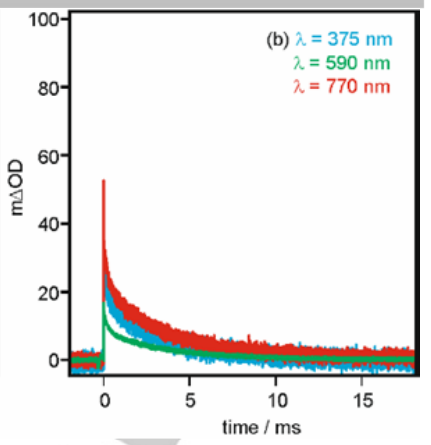

Figure 3. Decays of the transient absorption signals at different wavelengths on two different timescales. Measured after excitation of $20 \mu \mathrm{M}$ pentad I in deaerated $\mathrm{CH}_{3} \mathrm{CN}$ at $532 \mathrm{~nm}$. $50 \mathrm{mM} \mathrm{Sc}(\mathrm{OTf})_{3}$ was present.

Experiments to quantify the pre-association between $A Q$ and $\mathrm{Sc}^{3+}$ or $\mathrm{Ca}^{2+}$ by various experimental techniques (NMR, UV-Vis, IR) were unsuccessful. Prior studies demonstrated that this is possible for o-benzoquinones which can chelate small Lewis acids, but in the case of $p$-benzoquinones this pre-association generally seems to remain undetectable. ${ }^{[17-18]}$ Upon reduction to $A Q^{-}$and $A^{2-}$, the formation of Lewis acid - Lewis base adducts then becomes directly observable, for example by cyclic voltammetry (Figure S4, see below).

Under suitable conditions, two $\mathrm{Sc}^{3+}$ cations readily bind to the electrochemically generated dianionic form of 9,10anthraquinone (purple trace in Figure 2e), but in pentad I only one $\mathrm{Sc}^{3+}$ cation binds to the photochemically generated $\mathrm{AQ}^{2-}$ species. In pentad I, the $A Q$ unit is flanked by two phenylene bridging units bearing a total of $4 n$-hexyl substituents, and hence steric effects could make cation binding more unfavorable in this case, leading to 1:1 rather than 1:2 association between $\mathrm{AQ}^{2-}$ and $\mathrm{Sc}^{3+}$. Moreover, there is the possibility of electrostatic repulsion between $\mathrm{Sc}^{3+}$ and the adjacent $\mathrm{Ru}(\mathrm{bpy})_{3}{ }^{2+}$ units. The same effects can also explain the relatively slow adduct formation between $\mathrm{AQ}^{-}$and $\mathrm{Sc}^{3+}(170 \mathrm{~ns}$, blue trace in Figure 2a).

The transient absorption experiment in Figure 2 was restricted to a time window of $20 \mu \mathrm{s}$. On longer timescales no further spectral changes occur (Figure S6), and the remaining transient absorption signal (red trace in Figure 2a) decays only very slowly. Figure 3 contains single-wavelength kinetics, monitoring the decay of the signals at 375,590 , and $770 \mathrm{~nm}$ on micro- and millisecond timescales. These transients contain the initial 170 ns and $1.8 \mu$ s decay components identified above (particularly evident in the green trace in Figure 3a), and bi-exponential fits to the remaining slower decays yield lifetimes of $280 \mu \mathrm{s}$ and $3.6 \mathrm{~ms}$. On such slow timescales bimolecular encounters can obviously occur, ${ }^{[24 e]}$ and therefore it is unlikely that these decay times reflect intramolecular events. One of the most plausible bimolecular reactions which can account for the longevity of the transient absorption signals is electron self-exchange between $\mathrm{TAA}^{+}$and TAA, which leads to a spatial separation of oxidation and reduction products on separate pentad molecules (Scheme 3a). By means of UV-Vis transient absorption spectroscopy the resulting products are indistinguishable from the initial photoproduct containing $\mathrm{AQ}^{2-} / \mathrm{Sc}^{3+}$ and $2 \mathrm{TAA}^{+}$units on a given pentad. Several other bimolecular reaction pathways are conceivable (e. g., that in Scheme 3b), but the key point is that the charge accumulated state comprised of the $\mathrm{AQ}^{2-} / \mathrm{Sc}^{3+}$ 
adduct (initially formed in an intramolecular reaction) persists on a millisecond timescale.

(a)

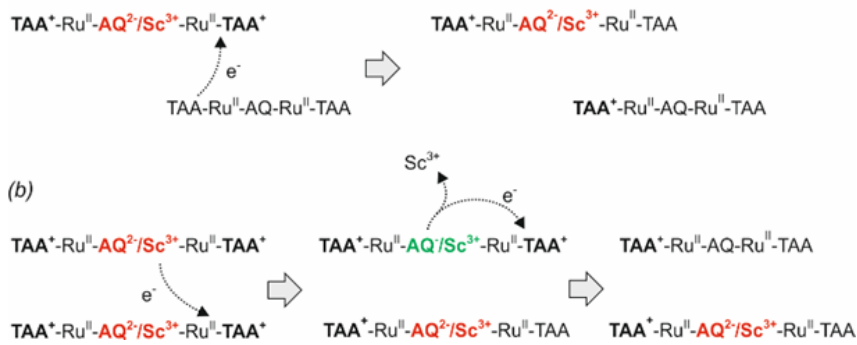

Scheme 3. Two possible bimolecular reaction sequences that can follow intramolecular photo-induced charge accumulation.

In order for bimolecular reactions to occur at appreciable rates between reactants present at $\mu \mathrm{M}$ concentrations, intramolecular charge separation between $\mathrm{AQ}^{2-} / \mathrm{Sc}^{3+}$ and the $2 \mathrm{TAA}^{+}$units is required to persist for at least a few microseconds. ${ }^{[6 f, 24 \mathrm{e}]}$ In our prior transient IR study, the $A Q^{2-}$ species exhibited a lifetime of 870 ns in neat de-aerated $\mathrm{CH}_{3} \mathrm{CN}$ at $20{ }^{\circ} \mathrm{C} .^{[14]}$ In the present study, the formation of an adduct with $\mathrm{Sc}^{3+}$ causes a substantial thermodynamic stabilization of the $\mathrm{AQ}^{2-}$ species (see below), and consequently the deceleration of intramolecular charge recombination to (at least) a few microseconds is not surprising. Cyclic voltammetry permits estimation of the thermodynamic stabilization of $A Q^{-}$and $\mathrm{AQ}^{2-}$ in presence of $\mathrm{Sc}^{3+}$. In neat deaerated $\mathrm{CH}_{3} \mathrm{CN}$, the $A Q^{-} / \mathrm{AQ}$ and $\mathrm{AQ}^{2-} / \mathrm{AQ}^{-}$redox couples appear at -0.91 and $-1.34 \mathrm{~V}$ vs. SCE, respectively (black trace in Figure 4). In presence of $50 \mathrm{mM} \mathrm{Ca}\left(\mathrm{ClO}_{4}\right)_{2}$ (blue trace) or $50 \mathrm{mM}$ $\mathrm{Sc}(\mathrm{OTf})_{3}$ (red trace) the two redox couples are no longer resolvable from one another, similar to what was previously reported for other quinones in presence of Lewis acids, ${ }^{[17-18]}$ or what is seen for $p$-benzoquinones in presence of Bronsted acids. ${ }^{[27]}$ The reason for this is that both types of acids have a stronger influence on the $A Q^{2-} / A Q^{-}$potential than on the $A Q^{-} / A Q$ couple, because binding to $A Q^{2-}$ is stronger than to $A Q^{-}$, at least in isolated 9,10-anthraquinone. The stronger potential shift observed for $\mathrm{Sc}^{3+}$ with respect to $\mathrm{Ca}^{2+}$ reflects the difference in Lewis acidity between these two cations. ${ }^{[17-18]}$ At the high cation concentrations used for the data in Figure $4(50 \mathrm{mM})$, the observable redox waves are quasi-reversible (see Figure S3 for concentration-dependent studies), and a potential of $0.38 \mathrm{~V}$ vs. $S C E$ is extracted for the two-electron reduction of $A Q$ to $A Q^{2-}$ in presence of $50 \mathrm{mM} \mathrm{Sc}^{3+}$. The electrochemical potential for oxidation of TAA to TAA ${ }^{+}$in pentad $\mathbf{I}$ is $0.69 \mathrm{~V}$ vs. SCE (Figure S5), ${ }^{[14]}$ and consequently one can estimate that the intramolecular charge-accumulated state comprised of the $\mathrm{AQ}^{2-}$, $\mathrm{Sc}^{3+}$ adduct and $2 \mathrm{TAA}^{+}$units is energetically ca. $2 \cdot e \cdot(0.69 \mathrm{~V}-$ $0.38 \mathrm{~V})=0.62 \mathrm{eV}$ above the ground state. For comparison, in absence of $\mathrm{Sc}^{3+}$ the respective charge-accumulated state stores $3.56 \mathrm{eV}^{[14]}$ Given the very strong stabilization of the chargeaccumulated state in presence of $\mathrm{Sc}^{3+}$, it is not surprising that intramolecular charge-recombination is slowed down to an extent that bimolecular reactions leading to the very slow decays in Figure 3 become possible. No further redox waves are detectable in the -1.5 to $0.8 \mathrm{~V}$ vs. SCE potential range.

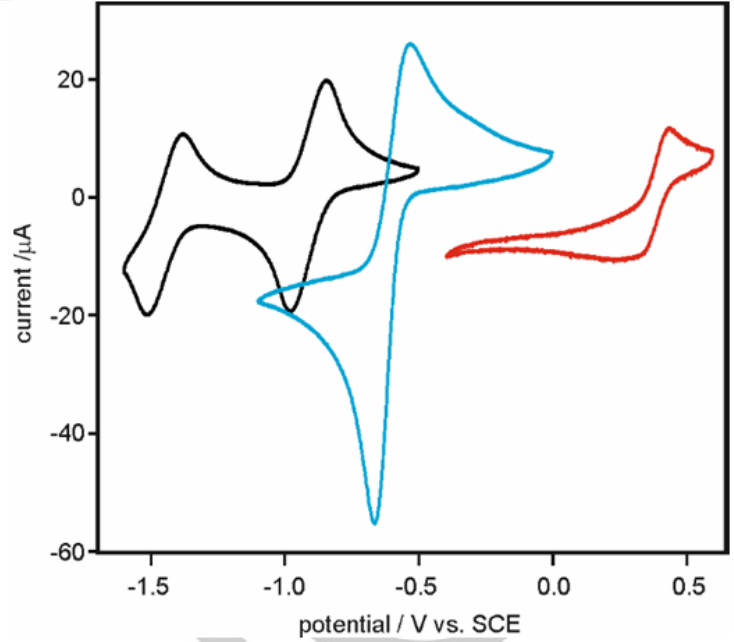

Figure 4. Cyclic voltammetry of 9,10-anthraquinone $(A Q)$ in dry de-aerated $\mathrm{CH}_{3} \mathrm{CN}$ containing $0.1 \mathrm{M} \mathrm{TBAPF}_{6}$. Black trace: neat solvent. Blue trace: in presence of $50 \mathrm{mM} \mathrm{Ca}\left(\mathrm{ClO}_{4}\right)_{2}$. Red trace: in presence of $50 \mathrm{mM} \mathrm{Sc}(\mathrm{OTf})_{3}$.

\section{Conclusions}

Exceptionally long-lived accumulation of electrons without sacrificial donors has been achieved through exploitation of Lewis acid - Lewis base interactions between $\mathrm{Sc}^{3+}$ and anthraquinone dianion, using visible light as the only energy input. Electron accumulation in pentad $\mathbf{I}$ is a reversible intramolecular process which relies on covalently attached donors, but the quinone dianion $/ \mathrm{Sc}^{3+}$ adduct is so long-lived that ensuing bimolecular reactions lead to a spatial separation of oxidative and reductive equivalents on separate molecules even at concentrations as low as $20 \mu \mathrm{M}$. The net result is a chargeaccumulated species with a lifetime in the millisecond regime, far exceeding the lifetime of $4.7 \mu$ s detected in presence of Bronsted acid. ${ }^{[16]}$ The lifetime determined here in presence of the Lewis acid $\mathrm{Sc}^{3+}$ is in fact comparable to what is observed for some of the intermediates of the Kok cycle. ${ }^{[19]}$ This finding is unprecedented for artificial molecular systems that do not rely on sacrificial reagents.

\section{Experimental Section}

The synthesis of pentad I has been reported previously. ${ }^{[14]}$ UV-vis absorption, cyclic voltammetry, spectro-electrochemistry, and nanosecond transient absorption spectroscopy was performed using equipment described previously. ${ }^{[6 f,}{ }^{24 \mathrm{e}]}$ For recording the transient absorption data that formed the basis for the determination of speciesassociated difference spectra (SADS), the so-called mapping option from Edinburgh Instruments was employed.

\section{Acknowledgements}

This work was funded by the Swiss National Science Foundation through grant number 200021_146231/1 and through the NCCR Molecular Systems Engineering.

Keywords: electron transfer $\cdot$ donor-acceptor systems $\cdot$ timeresolved spectroscopy $\bullet$ photochemistry $\bullet$ energy conversion 
[1] T. J. Meyer, M. H. V. Huynh, H. H. Thorp, Angew. Chem. Int. Ed. 2007, 46, 5284-5304.

[2] a) A. M. Appel, J. E. Bercaw, A. B. Bocarsly, H. Dobbek, D. L. DuBois, M. Dupuis, J. G. Ferry, E. Fujita, R. Hille, P. J. A. Kenis, C. A. Kerfeld R. H. Morris, C. H. F. Peden, A. R. Portis, S. W. Ragsdale, T. B. Rauchfuss, J. N. H. Reek, L. C. Seefeldt, R. K. Thauer, G. L. Waldrop Chem. Rev. 2013, 113, 6621-6658; b) X. Sala, S. Maji, R. Bofill, J. Garcia-Anton, L. Escriche, A. Llobet, Acc. Chem. Res. 2014, 47, 504516

[3] Y. Pellegrin, F. Odobel, C. R. Chimie 2017, 20, 283-295.

[4] a) M. R. Wasielewski, Chem. Rev. 1992, 92, 435-461; b) M. M. Waskasi, G. Kodis, A. L. Moore, T. A. Moore, D. Gust, D. V. Matyushov J. Am. Chem. Soc. 2016, 138, 9251-9257; c) M. Delor, T. Keane, P. A Scattergood, I. V. Sazanovich, G. M. Greetham, M. Towrie, A. Meijer, J A. Weinstein, Nature Chem. 2015, 7, 689-695; d) F. Zieschang, M. H Schreck, A. Schmiedel, M. Holzapfel, J. H. Klein, C. Walter, B. Engels, C. Lambert, J. Phys. Chem. C 2014, 118, 27698-27714; e) M. B Majewski, N. R. de Tacconi, F. M. MacDonnell, M. O. Wolf, Chem.-Eur J. 2013, 19, 8331-8341; f) S.-H. Lee, C. T.-L. Chan, K. M.-C. Wong, W H. Lam, W.-M. Kwok, V. W. W. Yam, J. Am. Chem. Soc. 2014, 136, 10041-10052

[5] a) M. R. Wasielewski, M. P. Niemczyk, W. A. Svec, E. B. Pewitt, J. Am. Chem. Soc. 1985, 107, 1080-1082; b) G. L. Closs, J. R. Miller, Science 1988, 240, 440-447; c) L. S. Fox, M. Kozik, J. R. Winkler, H. B. Gray Science 1990, 247, 1069-1071.

[6] a) W. B. Davis, W. A. Svec, M. A. Ratner, M. R. Wasielewski, Nature 1998, 396, 60-63; b) E. A. Weiss, M. J. Ahrens, L. E. Sinks, A. V. Gusev, M. A. Ratner, M. R. Wasielewski, J. Am. Chem. Soc. 2004, 126, 5577-5584; c) F. Giacalone, J. L. Segura, N. Martín, D. M. Guldi, J. Am Chem. Soc. 2004, 126, 5340-5341; d) P. P. Edwards, H. B. Gray, M. T. J. Lodge, R. J. P. Williams, Angew. Chem. Int. Ed. 2008, 47, 67586765; e) M. Cordes, B. Giese, Chem. Soc. Rev. 2009, 38, 892-901; f) M. Kuss-Petermann, O. S. Wenger, J. Am. Chem. Soc. 2016, 138, 1349 1358; g) C. Atienza-Castellanos, M. Wielopolski, D. M. Guldi, C. van der Pol, M. R. Bryce, S. Filippone, N. Martín, Chem. Commun. 2007, 5164-5166; h) N. Renaud, M. A. Harris, A. P. N. Singh, Y. A. Berlin, M A. Ratner, M. R. Wasielewski, F. D. Lewis, F. C. Grozema, Nature Chem. 2016, 8, 1015-1021

[7] a) J. Sukegawa, C. Schubert, X. Z. Zhu, H. Tsuji, D. M. Guldi, E. Nakamura, Nature Chem. 2014, 6, 899-905; b) M. P. Eng, B. Albinsson, Angew. Chem. Int. Ed. 2006, 45, 5626-5629; c) B. Albinsson, M. P. Eng K. Pettersson, M. U. Winters, Phys. Chem. Chem. Phys. 2007, 9, 58475864; d) C. Shih, A. K. Museth, M. Abrahamsson, A. M. BlancoRodriguez, A. J. Di Bilio, J. Sudhamsu, B. R. Crane, K. L. Ronayne, M. Towrie, A. Vlček, J. H. Richards, J. R. Winkler, H. B. Gray, Science 2008, 320, 1760-1762; e) H. A. Meylemans, J. T. Hewitt, M. Abdelhaq P. J. Vallett, N. H. Damrauer, J. Am. Chem. Soc. 2010, 132, 11464 11466; f) D. C. O'Hanlon, B. W. Cohen, D. B. Moravec, R. F. Dallinger M. D. Hopkins, J. Am. Chem. Soc. 2014, 136, 3127-3136; g) M. Natali, S. Campagna, F. Scandola, Chem. Soc. Rev. 2014, 43, 4005-4018.

[8] a) A. A. Pizano, J. L. Yang, D. G. Nocera, Chem. Sci. 2012, 3, $2457-$ 2461; b) A. Magnuson, H. Berglund, P. Korall, L. Hammarström, B. Åkermark, S. Styring, L. C. Sun, J. Am. Chem. Soc. 1997, 119, 1072010725; c) L. C. Sun, M. Burkitt, M. Tamm, M. K. Raymond, M. Abrahamsson, D. LeGourriérec, Y. Frapart, A. Magnuson, P. H. Kenéz, P. Brandt, A. Tran, L. Hammarström, S. Styring, B. Åkermark, J. Am. Chem. Soc. 1999, 121, 6834-6842; d) T. Irebo, S. Y. Reece, M. Sjödin, D. G. Nocera, L. Hammarström, J. Am. Chem. Soc. 2007, 129, 1546215464; e) J. Chen, M. Kuss-Petermann, O. S. Wenger, Chem. Eur. J. 2014, 20, 4098-4104; f) J. D. Megiatto, D. D. Mendez-Hernandez, M. E. Tejeda-Ferrari, A. L. Teillout, M. J. Llansola-Portoles, G. Kodis, O. G Poluektov, T. Rajh, V. Mujica, T. L. Groy, D. Gust, T. A. Moore, A. L. Moore, Nature Chem. 2014, 6, 423-428; g) N. H. Damrauer, J. M. Hodgkiss, J. Rosenthal, D. G. Nocera, J. Phys. Chem. B 2004, 108 6315-6321.

[9] a) L. Hammarström, Acc. Chem. Res. 2015, 48, 840-850; b) Y Pellegrin, F. Odobel, Coord. Chem. Rev. 2011, 255, 2578-2593; c) A. G. Bonn, O. S. Wenger, Chimia 2015, 69, 17-21.
[10] a) M. P. O'Neil, M. P. Niemczyk, W. A. Svec, D. Gosztola, G. L. Gaines, M. R. Wasielewski, Science 1992, 257, 63-65; b) H. Imahori, M. Hasegawa, S. Taniguchi, M. Aoki, T. Okada, Y. Sakata, Chem. Lett. 1998, 27, 721-722

[11] T. H. Ghaddar, J. F. Wishart, D. W. Thompson, J. K. Whitesell, M. A. Fox, J. Am. Chem. Soc. 2002, 124, 8285-8289.

[12] a) R. Konduri, H. W. Ye, F. M. MacDonnell, S. Serroni, S. Campagna, K. Rajeshwar, Angew. Chem. Int. Ed. 2002, 41, 3185-3187; b) R. Konduri, N. R. de Tacconi, K. Rajeshwar, F. M. MacDonnell, J. Am. Chem. Soc 2004, 126, 11621-11629; c) K. L. Wouters, N. R. de Tacconi, R. Konduri, R. O. Lezna, F. M. MacDonnell, Photosynth. Res. 2006, 87 41-55; d) G. F. Manbeck, K. J. Brewer, Coord. Chem. Rev. 2013, 257, 1660-1675; e) M. Elvington, K. J. Brewer, Inorg. Chem. 2006, 45, 5242 5244; f) M. Elvington, J. Brown, S. M. Arachchige, K. J. Brewer, J. Am. Chem. Soc. 2007, 129, 10644-10645; g) B. Matt, J. Fize, J. Moussa, H Amouri, A. Pereira, V. Artero, G. Izzet, A. Proust, Energy Environ. Sci. 2013, 6, 1504-1508; h) D. Polyansky, D. Cabelli, J. T. Muckerman, E. Fujita, T. Koizumi, T. Fukushima, T. Wada, K. Tanaka, Angew. Chem. Int. Ed. 2007, 46, 4169-4172; i) D. E. Polyansky, D. Cabelli, J. T. Muckerman, T. Fukushima, K. Tanaka, E. Fujita, Inorg. Chem. 2008, 47, 3958-3968; j) G. Knör, A. Vogler, S. Roffia, F. Paolucci, V. Balzani, Chem. Commun. 1996, 1643-1644; k) K. T. Oppelt, E. Woss, M. Stiftinger, W. Schofberger, W. Buchberger, G. Knör, Inorg. Chem. 2013, 52, 11910-11922; I) L. Zedler, S. Kupfer, I. R. de Moraes, M. Wächtler, R. Beckert, M. Schmitt, J. Popp, S. Rau, B. Dietzek, Chem.-Eur. J. 2014, 20, 3793-3799; m) K. Kitamoto, M. Ogawa, G. Ajayakumar, S. Masaoka, H. B. Kraatz, K. Sakai, Inorg. Chem. Front. 2016, 3, 671-680; n) K. Kitamoto, K. Sakai, Chem. Eur. J. 2016, 22, 12381-12390.

[13] a) S. Karlsson, J. Boixel, Y. Pellegrin, E. Blart, H. C. Becker, F. Odobel, L. Hammarström, Faraday Discuss. 2012, 155, 233-252; b) S. Karlsson, J. Boixel, Y. Pellegrin, E. Blart, H. C. Becker, F. Odobel, L. Hammarström, J. Am. Chem. Soc. 2010, 132, 17977-17979.

[14] M. Orazietti, M. Kuss-Petermann, P. Hamm, O. S. Wenger, Angew. Chem. Int. Ed. 2016, 55, 9407-9410.

[15] M. Kuss-Petermann, O. S. Wenger, Helv. Chim. Acta 2017, 100 e1600283.

[16] M. Kuss-Petermann, M. Orazietti, M. Neuburger, P. Hamm, O. S. Wenger, J. Am. Chem. Soc. 2017, 139, 5225-5232.

[17] a) S. Fukuzumi, Chem. Lett. 2008, 37, 808-813; b) S. Fukuzumi, K. Ohkubo, Coord. Chem. Rev. 2010, 254, 372-385; c) S. Fukuzumi, K Ohkubo, Y. Morimoto, Phys. Chem. Chem. Phys. 2012, 14, 8472-8484; d) Y. Morimoto, H. Kotani, J. Park, Y. M. Lee, W. Nam, S. Fukuzumi, J. Am. Chem. Soc. 2011, 133, 403-405; e) J. Park, Y. Morimoto, Y. M. Lee, W. Nam, S. Fukuzumi, J. Am. Chem. Soc. 2011, 133, 5236-5239; f) S. Fukuzumi, K. Ohkubo, Y. M. Lee, W. Nam, Chem. Eur. J. 2015, 21, 17548-17559.

[18] a) S. Fukuzumi, H. Mori, H. Imahori, T. Suenobu, Y. Araki, O. Ito, K. M. Kadish, J. Am. Chem. Soc. 2001, 123, 12458-12465; b) S. Fukuzumi, K. Okamoto, Y. Yoshida, H. Imahori, Y. Araki, O. Ito, J. Am. Chem. Soc. 2003, 125, 1007-1013; c) T. Kawashima, K. Ohkubo, S. Fukuzumi, Phys. Chem. Chem. Phys. 2011, 13, 3344-3352; d) A. G. Bonn, O. S. Wenger, Phys. Chem. Chem. Phys. 2015, 17, 24001-24010.

[19] a) B. Kok, B. Forbush, M. McGloin, Photochem. Photobiol. 1970, 11, 457-475; b) A. W. Rutherford, Trends Biochem. Sci. 1989, 14, 227-232.

[20] A. Babaei, P. A. Connor, A. J. McQuillan, S. Umapathy, J. Chem. Ed. 1997, 74, 1200-1204.

[21] H. Pal, T. Mukherjee, J. P. Mittal, J. Chem. Soc., Faraday Trans. 1994 90, 711-716.

[22] B. E. Hulme, G. O. Phillips, E. J. Land, J. Chem. Soc., Faraday Trans 1972, 68, 1992-2002.

[23] I. H. M. van Stokkum, D. S. Larsen, R. van Grondelle, Biochim. Biophys. Acta 2004, 1657, 82-104.

[24] a) S. L. Mecklenburg, D. G. McCafferty, J. R. Schoonover, B. M. Peek, B. W. Erickson, T. J. Meyer, Inorg. Chem. 1994, 33, 2974-2983; b) K. A. Opperman, S. L. Mecklenburg, T. J. Meyer, Inorg. Chem. 1994, 33, 5295-5301; c) J. Hankache, M. Niemi, H. Lemmetyinen, O. S. Wenger, Inorg. Chem. 2012, 51, 6333-6344; d) J. Hankache, O. S. Wenger, Chem. Eur. J. 2012, 18, 6443-6447; e) M. Kuss-Petermann, O. S. Wenger, Angew. Chem. Int. Ed. 2016, 55, 815-819. 
[25] K. Sreenath, T. G. Thomas, K. R. Gopidas, Org. Lett. 2011, 13, 1134 1137.

[26] J. Hankache, M. Niemi, H. Lemmetyinen, O. S. Wenger, J. Phys. Chem. A 2012, 116, 8159-8168.

[27] a) M. Quan, D. Sanchez, M. F. Wasylkiw, D. K. Smith, J. Am. Chem. Soc. 2007, 129, 12847-12856; b) N. Gupta, H. Linschitz, J. Am. Chem. Soc.

1997 ,

119 ,

6384-6391. 


\section{Entry for the Table of Contents}

\section{FULL PAPER}

Photoinduced charge accumulation is very difficult to achieve without sacrificial reagents. Metal ion-coupled electron transfer (MCET) is found to lead to an unusually longlived charge-accumulated state in a molecular pentad.

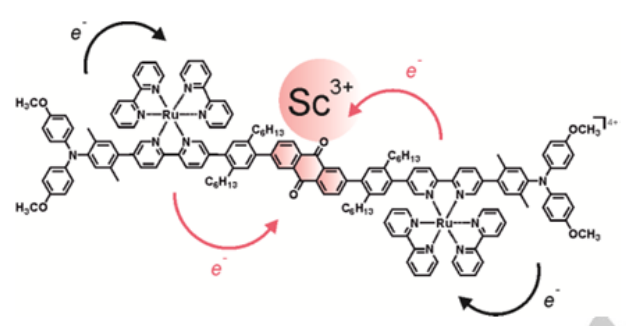

Martin Kuss-Petermann, Oliver S. Wenger*

Page No. - Page No.

Exceptionally Long-Lived Photodriven Multi-Electron Storage without Sacrificial Reagents 